\title{
$\underline{\mathbf{P}-95}$
}

\section{Comparative Antioxidant Activities of Soybean and Tempeh Extract in Rat Brain}

\author{
Siti Murnirah Jaafar ${ }^{1}$, Aliya Ahmad ${ }^{1}$, Kalavathy Ramasamy ${ }^{2}$ Abu Bakar Abdul Majeed ${ }^{1}$ and \\ Vasudevan Mani, ${ }^{1, *}$ \\ ${ }^{1}$ Brain Research Laboratory, Faculty of Pharmacy, UiTM Puncak Alam, 42300 Bandar Puncak Alam, Selangor Darul \\ Ehsan, Malaysia; ${ }^{2}$ Colloborative Drug Development and Research(CDDR) Group, Faculty of Pharmacy, UiTM Puncak \\ Alam, 42300 Bandar Puncak Alam, Selangor Darul Ehsan, Malaysia; E-mail:vasudevan@puncakalam.uitm.edu.my
}

Soybean and local fermented soybean, tempeh have been known for their health benefits and memory enhancement. It has been reported that soybean has potential role in reducing the progression of neurodegenerative disorder. Reactive oxygen species (ROS) play a crucial role in ageing and age-related neurodegenerative changes. The present study highlighted the comparative studies between total isoflavone extract from soybean and tempeh on the neuronal antioxidant. The total isoflavone extracts were administered orally for 15 days with three selected doses (10, 20 and $40 \mathrm{mg} / \mathrm{kg})$. Piracetam (400 mg/kg, p.o.) was used as standard drug. The measurement of brain catalase, superoxide dismutase (SOD), glutathione (GSH), glutathione reductase (GR), TBARS and nitric oxide (NO) were extended. Our results showed that soybean and tempeh extracts significantly improved antioxidant activities by significantly increased the levels of catalase, SOD, GR and GSH while decreased levels of TBARS and NO level in the rat brain. This suggests that soybean and tempeh extract might be useful in the age-related neurodegenerative changes including management and prevention of Alzheimer's disease through its antioxidant activities.

Keywords: Reactive oxygen species, isoflavone, antioxidant, neurodegeneration, Alzheimer's disease. 